\title{
États-Unis. Un nouvel élan attendu pour l'éducation publique
}

Jean-Pierre Véran

\section{OpenEdition}

\section{Journals}

Édition électronique

URL : https://journals.openedition.org/ries/6305

DOI : 10.4000/ries.6305

ISSN : 2261-4265

Éditeur

France Education international

Édition imprimée

Date de publication : 1 septembre 2018

Pagination : 15-16

ISBN : 978-2-85420-619-7

ISSN : $1254-4590$

Référence électronique

Jean-Pierre Véran, «États-Unis. Un nouvel élan attendu pour l'éducation publique », Revue

internationale d'éducation de Sèvres [En ligne], 78 I septembre 2018, mis en ligne le 01 septembre 2020, consulté le 25 juin 2021. URL : http://journals.openedition.org/ries/6305 ; DOI : https://doi.org/ $10.4000 /$ ries.6305

Ce document a été généré automatiquement le 25 juin 2021.

(C) Tous droits réservés 


\title{
États-Unis. Un nouvel élan attendu pour l'éducation publique
}

\author{
Jean-Pierre Véran
}

1 Si l'on en croit une note du Centre pour le budget et les priorités politiques publiée en novembre $2017^{1}$, intitulée Une décennie punitive pour le budget de l'éducation, douze États américains ont réduit le budget de l'éducation depuis la crise de 2008 et ont maintenu cette restriction, malgré l'embellie économique survenue depuis. Selon les auteurs, en 2015 (dernière année pour laquelle on dispose des chiffres concernés), 29 États consacraient moins qu'ils ne le faisaient en 2008 à la dépense par élève. Comme, en 2017, 22 États ont fait face à des déficits de rentrées budgétaires, la situation de l'école publique risque d'être fort sérieuse pour longtemps encore. Et ces coupes budgétaires risquent encore de diminuer la possibilité de développer l'intelligence et la créativité des travailleurs et entrepreneurs de demain. Selon les auteurs, la recherche comme le bon sens indiquent que ces restrictions du budget de l'éducation pénalisent prioritairement les enfants dont les familles sont en situation de pauvreté ou de précarité. Restaurer les budgets de l'éducation publique serait donc une priorité politique.

2 Les auteurs rejoignent ainsi les préoccupations exprimées en 2013 dans le rapport de la Commission pour l'équité et l'excellence intitulé For each and every child ${ }^{2}$, définissant une stratégie pour l'égalité et l'excellence en éducation.

3 Le système scolaire public aux États-Unis, pris dans son ensemble, manque à ses devoirs envers notre nation et nos enfants. Nos leaders dénoncent mais tolèrent les disparités entre les résultats des élèves, ce qui est injuste mais également dangereux socialement et économiquement.

4 Quelques mois après la publication de cette note, l'actualité du printemps 2018 a été marquée par des manifestations d'enseignants américains pour des revalorisations salariales. Ce mouvement, qui a mobilisé les enseignants des établissements publics, intervient après une grève de neuf jours en mars en Virginie occidentale, à la suite de laquelle les enseignants du primaire ont obtenu leur première hausse de salaire depuis 
quatre ans. Dans l'oklahoma, la gouverneure a accordé une augmentation comprise entre 15 et $18 \%$.

5 Ce mouvement revendicatif n'est pas sans rappeler la grève des enseignants de Chicago, en 2012, qui s'était terminée sur un donnant-donnant bien symptomatique: l'augmentation de salaire obtenue au terme de neuf jours de grève $(+17 \%$ sur quatre ans) avait été liée à la mise en œuvre d'un nouveau système d'évaluation des enseignants prenant en compte les résultats de leurs élèves. Cette prise en compte faisait partie des mesures permettant de bénéficier des subventions du programme fédéral « Course en tête» (Race to the top), destinées aux écoles scolarisant des élèves issus de milieux défavorisés.

Dans un article publié en avril 2017 dans cette revue ${ }^{3}$, Jon Snyder et A. Lin Goodwin ont souligné, dans l'enseignement public américain, au sujet de la formation des enseignants, des "tensions persistantes " sur les méthodes d'évaluation de la qualité des enseignants et de leur formation "qui nécessitent des équilibrages et des ajustements constants». Les deux universitaires en appellent à « une gouvernance démocratique fondée sur les faits (...) pour parvenir à un parcours enseignant qui soutienne le progrès et le développement des enseignants, tout en fournissant les opportunités d'apprentissage que méritent nos enfants et que le bien être de cette communauté exige ».

7 Là encore, l'actualité de 2018 apporte un élément nouveau. Dans sa lettre annuelle du 13 février $2018^{4}$, la fondation Bill et Melinda Gates évoque les résultats des milliards qu'elle a investis dans l'éducation aux États-Unis :

Malheureusement, s'il y a eu quelques progrès au cours des dix dernières années, les écoles publiques américaines n'atteignent toujours pas certains niveaux importants, surtout le taux d'achèvement des études universitaires. Et les statistiques sont encore pires pour les élèves et étudiants de milieux défavorisés [...] Nous avons aussi travaillé avec des académies scolaires de l'ensemble du pays pour les aider à améliorer la qualité de l'enseignement. Cette initiative a aidé les éducateurs à comprendre comment observer les enseignants, à noter leurs résultats de manière juste et à les critiquer de manière constructive [...] Quel a été l'impact du travail que nous avons mené concernant l'efficacité des professeurs dans le cadre de ces trois tests? Son effet sur l'apprentissage des élèves a été mitigé [...] Pour obtenir un changement significatif, il faut parvenir à un consensus entre toute une série de décideurs: les autorités publiques, les commissions scolaires, les administrateurs, les enseignants et les parents d'élèves.

8 Il est intéressant de voir Bill et Melinda Gates porter sur les politiques américaines en éducation un regard convergent avec celui du Centre pour le budget et les priorités politiques, comme avec celui de la Commission pour l'équité et l'excellence, et en appeler au consensus entre toute une série de décideurs, rejoignant ainsi le souhait d'une gouvernance démocratique formulé par Snyder et Goodwin. Ces éclairages complémentaires permettent sans doute de mieux comprendre que la question d'un nouvel élan pour l'éducation publique est clairement posée dans le débat public aux États-Unis. 


\section{NOTES}

1. https:// www.cbpp.org/research/state-budget-and-tax/a-punishing-decade-for-schoolfunding

2. https://www2.ed.gov/about/bdscomm/list/eec/equity-excellence-commission-report.pdf

3. Jon Snyder, A. Lin Godwin «Devenir enseignant aux États-Unis: politiques, normes et tensions ", Revue internationale d'éducation de Sèvres, $n^{\circ}$ 74, avril 2017, p. 57-68. En ligne [DOI : 10.4000/ries.5793].

4. https://www.gatesnotes.com/media/AL2018/PDFs/2018AnnualLetter-FR.pdf

\section{INDEX}

Index géographique : États-Unis

Palabras claves : presupuesto educativo, gasto público, salario del profesor

Keywords : educational budgets, public expenditure, teacher wages

Mots-clés : budget de l'éducation, dépenses publiques, salaire des enseignants

\section{AUTEUR}

\section{JEAN-PIERRE VÉRAN}

Jean-Pierre Véran est inspecteur d'académie $(\mathrm{H})$, membre du comité de rédaction de la Revue internationale de Sèvres et expert auprès du CIEP en coopération éducative. Intervenant sur la gouvernance des organisations éducatives, les politiques éducatives et l'éducation aux médias et à l'information, il tient un blog consacré à l'éducation sur Mediapart : http://blogs.mediapart.fr/ blog/jean-pierre-veran/ Courriel : jeanpierreveran2@gmail.com. 